\title{
Influência dos fatores psíquicos e emocionais negativos no surgimento de doenças cardiovasculares: uma revisão de literatura
}

\author{
Influence of negative psychological and emotional factors on the onset of cardiovascular \\ diseases: a literature review
}

\author{
Influencia de factores psicológicos y emocionales negativos en la aparición de \\ enfermedades cardiovasculares: revisión de la literatura
}

Victor Carneiro de Souza ${ }^{1 *}$, Walter Oliveira Rios Junior ${ }^{1}$, Gabriel Magalhães Santos ${ }^{2}$, Sarah Araujo Lima $^{2}$, Natália Coelho Chester ${ }^{1}$, Mikaelle Lopes Rodrigues ${ }^{1}$, Camila de Oliveira Gregório ${ }^{1}$, Marcos Vinícios Marinho Gonçalves Franco ${ }^{1}$, Matheus de Vasconcelos Araújo ${ }^{3}$, Taissa Braga da Silva ${ }^{4}$.

\section{RESUMO}

Objetivo: Analisar a influência das doenças mentais, do estresse emocional e de outros fatores psíquicos e emocionais negativos no surgimento de disfunções cardiovasculares, em relação aos aspectos fisiopatológicos e de prevenção e tratamento. Métodos: Trata-se de um estudo de revisão integrativa. A busca de artigos foi realizada nas bases de dados da SciELO, LILACS e PubMed por meio dos descritores: "Doenças Cardiovasculares", "Doença das Coronárias", "Hipertensão", "Saúde Mental" e "Estresse Psicológico". Foram incluídos artigos em Português, Inglês e Espanhol publicados no período de 2002 a 2021 que atendessem ao objetivo do estudo. Resultados: Os principais mecanismos fisiopatológicos evidenciados foram: excesso de catecolaminas por hiperativação simpática, ativação do sistema renina-angiotensina-aldosterona, distúrbios da homeostase, estímulo à aterosclerose e baixa adesão a tratamentos medicamentosos. As principais intervenções preventivas e terapêuticas identificadas foram: prática de exercícios físicos aeróbicos e mudança nos hábitos alimentares. Ademais, a carga horária de trabalho e o sexo surgiram como fatores adicionais. Considerações finais: A identificação de mecanismos fisiopatológicos, preventivos e terapêuticos permite a intervenção adequada nesses pacientes a fim de evitar o desenvolvimento de doenças cardiovasculares. Assim, a prática de exercícios físicos e a melhora dos hábitos alimentares devem ser estimulados.

Palavras-chave: Saúde mental, Estresse psicológico, Doenças cardiovasculares.

\begin{abstract}
Objective: To analyze the influence of mental illness, emotional stress and other negative psychological and emotional factors in the appearance of cardiovascular disorders, in relation to the pathophysiological aspects and prevention and treatment. Methods: This is an integrative review study. The search for articles was carried out in the databases of SciELO, LILACS and PubMed through the descriptors: "Cardiovascular Diseases", "Coronary Disease", "Hypertension", "Mental Health" and "Psychological Stress". Articles in Portuguese, English and Spanish published from 2019 to 2021 that met the objective of the study were included. Results: The main pathophysiological mechanisms evidenced were: excess of catecholamines due to sympathetic hyperactivation, activation of the renin-angiotensin-aldosterone system, disturbances of homeostasis, stimulation of atherosclerosis and low adherence to drug treatments. The main preventive and therapeutic interventions identified were: the practice of aerobic physical exercises and changes in eating habits. In addition, workload and sex emerged as additional factors. Final considerations: The identification of pathophysiological, preventive and therapeutic mechanisms allows an adequate intervention in these patients in order to avoid the development of cardiovascular diseases. Thus, the practice of physical exercises and the improvement of eating habits should be encouraged.
\end{abstract}

Keywords: Mental health, Psychological stress, Cardiovascular diseases.

\section{RESUMEN}

Objetivo: Analizar la influencia de las enfermedades mentales, el estrés emocional y otros factores psicológicos y emocionales negativos en la aparición de trastornos cardiovasculares, en relación con los aspectos fisiopatológicos y de prevención y tratamiento. Métodos: Este es un estudio de revisión integradora.

\footnotetext{
${ }^{1}$ Universidade Federal do Ceará (UFC), Sobral - CE. *E-mail: victor_cds@yahoo.com.br

2 Universidade Federal do Ceará (UFC), Fortaleza - CE.

3 Universidade Estadual do Ceará (UECE), Fortaleza - CE.

${ }^{4}$ Centro Universitário INTA (UNINTA), Sobral - CE.
} 
La búsqueda de artículos se realizó en las bases de datos SciELO, LILACS y PubMed utilizando los descriptores: "Enfermedades cardiovasculares", "Enfermedad coronaria", "Hipertensión arterial", "Salud mental" y "Estrés psicológico". Se incluyeron artículos en portugués, inglés y español publicados de 2019 a 2021 que cumplieron con el objetivo del estudio. Resultados: Los principales mecanismos fisiopatológicos evidenciados fueron: exceso de catecolaminas por hiperactivación simpática, activación del sistema reninaangiotensina-aldosterona, alteraciones de la homeostasis, estimulación de la aterosclerosis y baja adherencia a los tratamientos farmacológicos. Las principales intervenciones preventivas y terapéuticas identificadas fueron: la práctica de ejercicios físicos aeróbicos y cambios en los hábitos alimentarios. Además, la carga de trabajo y el sexo surgieron como factores adicionales. Consideraciones finales: La identificación de mecanismos fisiopatológicos, preventivos y terapéuticos permite una adecuada intervención en estos pacientes para evitar el desarrollo de enfermedades cardiovasculares. Por tanto, conviene fomentar la práctica de ejercicios físicos y la mejora de los hábitos alimentarios.

Palabra clave: Salud mental, Estrés psicológico, Enfermedades cardiovasculares.

\section{INTRODUÇÃO}

As doenças cardiovasculares representam as principais causas de mortes no Brasil e no Mundo. De acordo com dados coletados no Sistema de Informação sobre Mortalidade (SIM), por ano, cerca de 300 mil pessoas sofrem Infarto Agudo do Miocárdio (IAM), ocorrendo óbito em 30\% desses casos. Estimativas apontam que, até 2040, haverá um aumento de aproximadamente 250\% desses eventos no Brasil (BRASIL, 2020).

Existem alguns fatores de risco já comprovados que influenciam no surgimento de doenças cardiovasculares por meio da aterosclerose, da hipertensão arterial e do desequilíbrio na produção de hormônios, tais como idade, sobrepeso, sedentarismo, ingesta excessiva de sal, alimentação rica em lipídeos, menopausa e fatores genéticos (DALMAZO AL, et al., 2019).

Entretanto, outros fatores podem também influenciar, tal qual a cultura popular há muito associa o estresse agudo e crônico com o desenvolvimento de doenças, o que culminou no surgimento de vários estudos epidemiológicos e alguns experimentais para verificar a ligação entre estresse mental e surgimento de diversas doenças, entre elas as cardiovasculares. Entretanto, muitos profissionais ainda enfrentam essa associação com ceticismo, desvalorizando a prática clínica investigativa por deixar de lado a pesquisa de outros fatores que poderiam predispor a patologias futuras (KOMORI T, et al., 2019).

Pessoas com depressão crônica possuem, cerca de duas vezes mais chance de desenvolver doenças cardiovasculares em um período de seis anos, tendo uma relevância negativa maior que a ansiedade. Apesar disso, as taxas de identificação e tratamento na atenção primária ainda são muito baixas, mesmo com grande relevância, comprovada pela prevalência de depressão e ansiedade em pessoas já com doenças cardiovasculares em 4 grandes cidades brasileiras de, respectivamente, 22 a $30 \%$ e 36 a 43\% (VELOSO MAOS, et al., 2019).

Mesmo com esses dados preocupantes, a saúde mental se apresenta como uma das áreas mais negligenciadas da saúde pública. Segundo a Organização Mundial da Saúde, quase 1 bilhão de pessoas vivem com transtorno mental no mundo. No atual contexto da pandemia da doença do coronavírus 2019 (COVID-19), esse quadro tende a se agravar ainda mais (OMS, 2020).

Uma vez destacado que situações estressantes podem levar, cronicamente, a desfechos cardiovasculares negativos, existem várias vias fisiológicas de ação, como na estimulação hipofisária, que levará a liberação de catecolaminas, na ativação do sistema renina-angiotensina-aldosterona, modificando os mecanismos pressóricos, e na privação do sono, que altera a produção de melatonina. Essas vias de ação alteradas causam diversos resultados secundários, como déficit do sistema imunológico e de coagulação, surgimento de hipertensão e remodelamento cardíaco (ALBUS C, et al., 2019).

Ademais, esse efeito do estresse pode causar modificações alimentares que irão interferir no metabolismo de vários nutrientes, tais como vitaminas do complexo $B$, vitamina $C$, cálcio, magnésio, ferro e zinco. Cronicamente, o sistema endócrino é afetado e pode gerar alterações no metabolismo de lipídeos, da pressão arterial e da frequência cardíaca, o que aumenta o consumo de oxigênio pelo miocárdio. Ao longo dos anos, essa situação pode favorecer o surgimento de hipertensão arterial sistêmica pela tensão desenvolvida nesses constantes momentos de ansiedade e estresse (DALMAZO AL, et al., 2019). 
Em meio a esse panorama, alguns esforços podem ser tomados para coibir a evolução dessas patologias, como a maior investigação clínica por parte de médicos, buscando verificar as fragilidades dos pacientes e analisando os prejuízos futuros se não houver auxílio adequado. Além disso, o exercício físico mostra excelentes resultados na redução tanto do risco cardiovascular quanto dos sintomas depressivos isoladamente (CASSIANO AN, et al., 2020).

Dessa forma, o estudo tem como objetivo analisar a relação entre o surgimento de doenças cardiovasculares com a presença de fatores psíquicos e emocionais negativos, através da análise de aspectos fisiopatológicos e estratégias de prevenção e tratamento.

\section{MÉTODOS}

Trata-se de um estudo desenvolvido segundo os critérios de revisão integrativa da literatura científica, a fim de determinar os efeitos que fatores psíquicos e emocionais negativos poderiam causar em um possível surgimento de doença cardiovascular.

A pergunta adotada para o planejamento desta pesquisa foi: os fatores psicológicos e emocionais cotidianos, em uma visão a médio e longo prazo, poderiam causar modificações orgânicas que culminariam no surgimento de doenças cardiovasculares e essas mudanças poderiam ser amenizadas?

Foi realizada uma pesquisa bibliográfica a fim de selecionar artigos que abordassem o tema proposto. Para tanto, foram utilizadas as bases de dados The Scientific Electronic Library Online (SciELO), Literatura Latino-americana e do Caribe em Ciências da Saúde (LILACS), US National Library of Medicine National Institutes of Health (Medline/PubMed).

A pesquisa foi realizada por meio da combinação de palavras-chaves indexadas nos Descritores em Ciência da Saúde (DeCS): "Doenças Cardiovasculares", "Doença das Coronárias", "Hipertensão", "Saúde Mental" e "Estresse Psicológico". Os descritores foram combinados utilizando os operadores booleanos "AND" e "OR". Foram utilizados artigos escritos em inglês, português, espanhol.

Foram excluídos trabalhos de revisão de literatura, resumos, teses e trabalhos que não possuíam os termos utilizados na busca como objeto principal. Inicialmente, 383 artigos foram encontrados. Uma triagem inicial, através da leitura dos títulos e resumos, foi realizada. Foram excluídos 47 artigos duplicados e 302 artigos que não se adequaram à temática estudada. Portanto, 17 foram selecionados para a análise (Figura 1).

Figura 1 - Fluxograma da aplicação dos critérios de inclusão e exclusão.

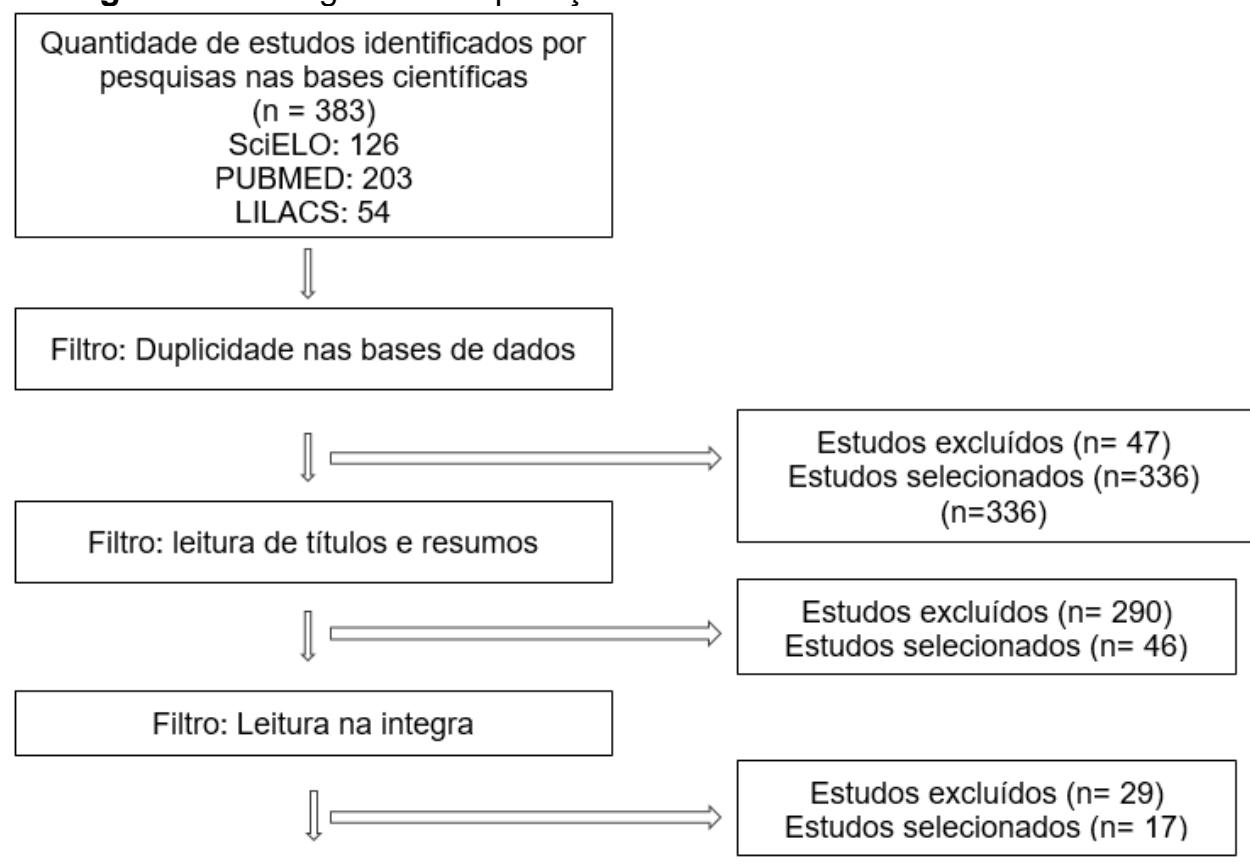

Fonte: Souza VC, et al., 2021. 
Em relação aos aspectos éticos, o estudo se adequa à resolução 466/12 do Conselho Nacional de Saúde (CNS). A análise foi realizada com artigos disponibilizados em Bases de Dados, acessíveis através da rede mundial de computadores. Por essa razão, conforme descreve a resolução, é dispensada a apreciação por um Comitê de Ética e Pesquisa (CEP). Além disso, o estudo zela por seus princípios de não maleficência, beneficência, autonomia e justiça.

\section{RESULTADOS}

A partir da aplicabilidade dos critérios de inclusão e exclusão, os resultados presentes neste trabalho abordam os principais achados dos dezessete estudos selecionados que estão expostos a seguir (Quadro 1).

Quadro 1 - Síntese dos principais achados sobre a relação entre fatores psíquicos e emocionais sobre o desenvolvimento de doenças cardiovasculares.

\begin{tabular}{|c|c|c|}
\hline $\mathbf{N}$ & Autores (Ano) & Principais achados \\
\hline 1 & ALMEIDA OP, et al. (2019) & $\begin{array}{l}\text { Estudo de coorte. A depressão está associada a um estilo de vida com } \\
\text { práticas que aumentam o risco de eventos cardiovasculares, além de } \\
\text { alterações na fisiologia. }\end{array}$ \\
\hline 2 & ANTUNES J (2020) & $\begin{array}{l}\text { Artigo de revisão. Os fatores relacionados ao ambiente de trabalho têm } \\
\text { forte influência sobre o desenvolvimento de doenças cardiovasculares. }\end{array}$ \\
\hline 3 & BUCCIARELLI V, et al. (2020) & $\begin{array}{l}\text { Artigo de revisão. A depressão se relaciona a sedentarismo, privação } \\
\text { de sono, alcoolismo e tabagismo e alterações orgânicas que predispõe } \\
\text { doenças cardiovasculares. }\end{array}$ \\
\hline 4 & CASSIANO AN, et al. (2020) & $\begin{array}{l}\text { Estudo de coorte. O exercício físico reduz o risco de doenças } \\
\text { cardiovasculares em pacientes depressivos. }\end{array}$ \\
\hline 5 & CHRISTIAN TMD (2015) & $\begin{array}{l}\text { Estudo de coorte. Um terço dos casos de Cardiomiopatia de Takotsubo } \\
\text { é desencadeado por fatores psicológicos. }\end{array}$ \\
\hline 6 & COUTO HA, et al. (2007) & $\begin{array}{l}\text { Artigo de revisão. O nível pressórico, devido a fatores estressantes e } \\
\text { ansiosos, se eleva no ambiente de trabalho. }\end{array}$ \\
\hline 7 & FROMMEYER G, et al. (2013) & $\begin{array}{l}\text { Artigo de revisão. A taquicardia supraventricular paroxística e o ataque } \\
\text { de pânico estão intimamente relacionados. }\end{array}$ \\
\hline 8 & GHADRI JR, et al. (2018) & $\begin{array}{l}\text { Artigo de revisão. O uso de medicamentos antidepressivos inibidores } \\
\text { da recaptação de serotonina e noradrenalina possivelmente } \\
\text { aumentam o risco de desenvolvimento de doenças cardiovasculares. }\end{array}$ \\
\hline 9 & JEE YH, et al. (2019) & $\begin{array}{l}\text { Estudo de coorte. O risco de doenças cardiovasculares é cerca de } 40 \% \\
\text { maior nos pacientes com depressão. }\end{array}$ \\
\hline 10 & LAMPERT R (2016) & $\begin{array}{l}\text { Artigo de revisão. Distúrbios psicológicos como depressão e ansiedade } \\
\text { aumentam a vulnerabilidade para taquiarritmias ventriculares em } \\
\text { cardiopatas. }\end{array}$ \\
\hline 11 & LARKIN E e WHOOLEY MA (2013) & $\begin{array}{l}\text { Artigo de revisão. Fatores psicológicos estão relacionados ao aumento } \\
\text { de biomarcadores inflamatórios e agregação plaquetária. Inibidores da } \\
\text { recaptação de serotonina seriam úteis. }\end{array}$ \\
\hline 12 & LOURES DL, et al. (2002) & $\begin{array}{l}\text { Artigo de revisão. O estresse crônico estimula a atividade do sistema } \\
\text { renina-angiotensina-aldosterona e causa alterações metabólicas e } \\
\text { vasculares que predispõem a aterosclerose. }\end{array}$ \\
\hline 13 & MENDES F e MARTINS D (2020) & $\begin{array}{l}\text { Artigo de revisão. O treinamento aeróbico de intensidade moderada } \\
\text { reduziu o modelamento cardíaco ventricular em testes com animais. }\end{array}$ \\
\hline 14 & RAJAN S, et al. (2020) & $\begin{array}{l}\text { Estudo de coorte. O risco de desenvolvimento de doença } \\
\text { cardiovascular foi } 14 \% \text { maior em indivíduos que apresentavam } 4 \text { ou } \\
\text { mais sintomas depressivos }\end{array}$ \\
\hline 15 & VELOSO MAOS, et al. (2019) & $\begin{array}{l}\text { Estudo transversal. O risco de desenvolver dislipidemia é maior entre } \\
\text { indivíduos ansiosos e depressivos. }\end{array}$ \\
\hline 16 & SCHMIDT K, et al. (2020) & $\begin{array}{l}\text { Estudo transversal. O sexo feminino é mais suscetível ao estresse } \\
\text { psicossocial, sendo mais propensas a patologias como cardiopatias e } \\
\text { doenças vasculares. }\end{array}$ \\
\hline 17 & STEVEN MB e JOHN SR (2015) & $\begin{array}{l}\text { Artigo de revisão. A depressão está relacionada à hipercortisolemia, } \\
\text { pacientes tendem a aderir mal à medicação e hábitos saudáveis. }\end{array}$ \\
\hline
\end{tabular}

Fonte: Souza VC, et al., 2021.

\section{Relação entre estresse psicológico e o desenvolvimento de doenças cardiovasculares}

O estresse psicológico pode ser definido como um quadro de desgaste mental, decorrente de uma exposição contínua do indivíduo a um grau de tensão desproporcional à capacidade de suportá-la, ou seja, 
entre as exigências impostas no cotidiano e aos meios do indivíduo para lidar com elas. No contexto clínico, o paciente pode apresentar, com intensidade relacionada aos fatores estressantes vividos, irritabilidade, dor na musculatura cervical, nervosismo, cefaleia por tensão, palpitações, fadiga, alteração do sono e períodos de indisposição (COUTO HA, et al., 2007).

Estudos demonstram que os valores aferidos de pressão arterial em ambientes de trabalho normalmente são mais elevados do que as obtidas em casa, devido a fatores estressantes e ansiosos, estando relacionados a uma possível hipertrofia ventricular esquerda, a qual cronicamente pode mudar a configuração cardíaca e gerar cardiopatias (COUTO HA, et al., 2007).

O estresse crônico estimula a atividade do sistema renina-angiotensina-aldosterona (SRAA), por meio de uma maior expressão renal de renina, bem como aumento de sua atividade plasmática pela ativação simpática. Portanto o consequente aumento dos níveis de angiotensina II, e consequentemente da aldosterona, surge como resposta adaptativa do organismo ao estresse mental. Essas substâncias irão agir no corpo por meio da retenção de água e sódio, da vasoconstrição, e do aumento de substâncias inflamatórias. Tais mecanismos estão diretamente relacionados ao surgimento de hipertensão arterial e remodelamento cardíaco (LOURES DL, et al., 2002).

O estresse mental também tem importante influência no desenvolvimento de isquemia miocárdica. Cronicamente, gera alterações metabólicas e vasculares, principalmente plaquetárias e lipídicas, que predispõem a aterogênese. De forma aguda, eleva o tônus vasomotor das coronárias, com redução do seu fluxo, e promove uma hiperatividade simpática resultando em aumento da frequência cardíaca, da pressão arterial sistêmica e na contratilidade miocárdica, aumentando a demanda do miocárdio por oxigênio e promovendo eventos isquêmicos (LOURES DL, et al., 2002).

\section{Relação entre depressão e doenças cardiovasculares}

A relação fisiopatológica entre depressão e risco cardiovascular foi alvo de diversos estudos. Indivíduos deprimidos geralmente adotam hábitos de vida que já são sabidamente fatores de risco cardiovascular, como sedentarismo, privação de sono, alcoolismo e tabagismo. Além disso, costumam ter baixa adesão a tratamentos farmacológicos (BUCCIARELLI V, et al., 2020).

Rajan S, et al. (2020) realizou um estudo prospectivo com 145.862 adultos de meia idade de diversos países economicamente distintos. O risco de desenvolvimento de doença cardiovascular foi $14 \%$ maior em indivíduos que apresentavam 4 ou mais sintomas depressivos. O risco era duas vezes maior nas áreas urbanas do que nas rurais. Além disso, indivíduos do sexo masculino apresentavam mais que o dobro de risco para o desenvolvimento de doenças cardiovasculares em comparação com as mulheres. A principal doença identificada foi o infarto do miocárdio.

O predomínio de casos em áreas urbanas pode estar relacionado a fatores que afetam a saúde mental desse grupo, como moradias superlotadas, escassez de áreas verdes e desigualdade social. Já o predomínio no sexo masculino, pode estar relacionado ao fato desse grupo procurar menos atendimento e apresentar dificuldade para relatar sintomas depressivos em comparação com as mulheres (RAJAN S, et al., 2020).

O risco de doenças cardiovasculares é cerca de $40 \%$ maior nos pacientes com depressão. Além disso, nos homens, esse distúrbio psicológico causou o aumento do risco de angina pectoris e de infarto agudo do miocárdio em até duas vezes. Fatores psicológicos, como o humor deprimido, aumentam em até 4 vezes a probabilidade de má adesão à medicação, além de não seguir recomendações de mudanças para um estilo de vida mais saudável. Refere ainda, uma maior inatividade física, predispondo a piores desfechos cardiovasculares (JEE YH, et al., 2019; STEVEN MB e JOHN SR, 2015).

Por outro lado, os próprios fatores psicossociais podem afetar diversas etapas orgânicas que resultam em aterotrombose, fato já identificado através da mensuração de biomarcadores circulantes de disfunção endotelial em pacientes depressivos. As alterações de hemostase e trombogênese desencadeados pelo estresse mental podem estar relacionados a uma redução da atividade do ativador do plasminogênio tecidual (tPA) e ao aumento do tempo de lise do coágulo e do inibidor do plasminogênio tecidual (LOURES DL, et al., 2002; BUCCIARELLI V, et al., 2020). 
Almeida OP, et al. (2019) segue essa linha de raciocínio ao afirmar que a depressão está diretamente associada a um estilo de vida com práticas que aumentam o risco de eventos cardiovasculares, além de alterações na fisiologia como redução da variabilidade da frequência cardíaca, aumento dos níveis basais de catecolaminas e de marcadores inflamatórios, além de disfunção endotelial e plaquetária.

Biomarcadores inflamatórios como proteína C reativa, interleucina 1 e interleucina 6, comumente associados ao desenvolvimento de aterosclerose, estavam em níveis aumentados em pacientes depressivos, tanto nos indivíduos saudáveis, quanto naqueles com alguma cardiopatia. (LARKIN E e WHOOLEY MA, 2013).

Uma relação íntima entre transtornos psiquiátricos e obesidade também já foi encontrada. Alguns estudos evidenciaram maior risco de dislipidemia entre indivíduos ansiosos e depressivos. Tal relação pode estar relacionada a um processo de inflamação crônica leve mediada por cortisol e desencadeada por alterações morfológicas no hipocampo presentes em pacientes com transtornos de humor. Alguns estudos já demonstraram a elevação dos níveis glicêmicos e o aumento da resistência à insulina, por mecanismos desconhecidos, durante situações de estresse (VELOSO MAOS, et al., 2019; LOURES DL, et al., 2002).

Steven MB e John SR (2015) também descrevem a hipercortisolemia como um dos fatores deflagrados pelos transtornos psiquiátricos relacionados ao aumento do risco cardiovascular. Segundo ele, esses distúrbios podem promover uma hiperatividade do eixo hipotálamo-hipófise-adrenal e defeitos na sinalização de serotonina, com consequente disfunção da amígdala. Associado à hipercortisolemia, a disfunção autonômica, o aumento de catecolaminas e de marcadores inflamatórios, além da disfunção endotelial e da ativação plaquetária contribuíram na fisiopatogênese do quadro.

Acerca da prevalência entre sexos, foi comprovado que homens possuem maior atividade autonômica cardíaca simpática, enquanto as mulheres, parassimpática. Durante o sono profundo e restaurador, acontece ativação vagal parassimpática e inibição simpática. Esses eventos são necessários para a redução fisiológica da pressão arterial e frequência cardíaca. Entretanto, em mulheres deprimidas, o sono é curto e pouco renovador, contribuindo para a hipersecreção de cortisol e impedindo a modulação da pressão arterial e da frequência cardíaca. Portanto, além de fatores como idade e obesidade, a modulação adrenérgica durante a situação de estresse é mais prevalente em mulheres pelo maior desequilíbrio das funções autonômicas (BUCCIARELLI V, et al., 2020).

\section{Aspectos laborais e o desenvolvimento de doenças cardiovasculares}

Em relação aos aspectos laborais, estudos demonstram que fatores relacionados ao ambiente de trabalho têm forte influência sobre o desenvolvimento de doenças cardiovasculares. Existem evidências sobre a excessiva ativação do sistema simpático-adrenérgico nos casos de longas jornadas de trabalho e horas extraordinárias em casa, principalmente quando há diminuição do tempo de recuperação. Esse tipo de rotina comumente está relacionado à inatividade física, ao tabagismo, à privação do sono, ao estresse psicológico, à obesidade e ao alcoolismo. $\mathrm{O}$ risco de infarto agudo do miocárdio aumenta substancialmente com 0 crescimento do número de horas semanais de trabalho (ANTUNES J, 2020).

O sexo feminino é mais suscetível ao estresse psicossocial devido à múltipla jornada de trabalho nos papeis sociais e familiares, sendo mais propensas a patologias como cardiopatias e doenças vasculares, evidenciado pelo infarto agudo do miocárdio e acidente vascular encefálico serem as principais causas de morte em mulheres com mais de 50 anos. Em contrapartida, apesar desses dados, o estresse em relação à doença isquêmica em específico apresenta mais desfechos negativos em homens. (SCHMIDT K, et al., 2020).

\section{Relação entre arritmias e saúde mental}

Diversos estudos evidenciaram o estresse como fator desencadeador de arritmias ventriculares e atriais, em razão da sua atuação através de alterações na atividade autonômica no indivíduo. Além disso, distúrbios psicológicos como depressão e ansiedade aumentam, de forma significativa, a vulnerabilidade para taquiarritmias ventriculares em cardiopatas. O estresse agudo ou grave pode, ainda, desencadear uma taquicardia ventricular mesmo sem uma cardiopatia de base (LAMPERT R, 2016). 
Também analisou a associação entre fatores psicossociais e arritmias. A taquicardia supraventricular paroxística (TSVP) e o Ataque de Pânico possuem uma apresentação clínica semelhante. A relação causa e consequência é difícil de ser estabelecida, pois muitas vezes os quadros não são documentos por análise eletrocardiográfica. A cura de pacientes com transtorno do pânico após ablação por cateter já observada em alguns estudos pode apontar que a TVSP é uma causa e não uma consequência de um ataque de pânico (FROMMEYER G, et al., 2013).

A correlação entre estresse mental e arritmias tem sua gênese na instabilidade elétrica miocárdica produzida pela hiperatividade simpática e pela redução da atividade parassimpática, o que poderia predispor o desenvolvimento de arritmias ventriculares graves. Além disso, a presença de isquemia agiria de forma sinérgica para o desenvolvimento do quadro (LOURES DL, et al., 2002).

\section{Cardiomiopatia de Takotsubo}

A Cardiomiopatia de Takotsubo (ou estresse) é uma doença intimamente ligada à fatores psicossociais, na qual um terço dos casos são desencadeados por fatores psicológicos, sendo descrita como "síndrome do coração partido" e, em casos menos comuns, como "síndrome do coração feliz" (CHRISTIAN TMD, 2015).

O mecanismo fisiopatológico desta doença envolve a liberação exacerbada de catecolaminas, o prejuízo na recaptação neuronal de norepinefrina e o estresse oxidativo, em resposta à situações de forte emoção, resultando em ações citotóxicas e comprometimento da microcirculação no miocárdio ventricular. Com base nisso, o uso de medicamentos antidepressivos inibidores da recaptação de serotonina e noradrenalina possivelmente aumentariam o risco desta doença (GHADRI JR, et al., 2018).

\section{Estratégias de prevenção e tratamento}

Cassiano AN, et al. (2020) realizou uma pesquisa com o objetivo de avaliar os efeitos da atividade física sobre o risco cardiovascular e a presença de sintomas depressivos em idosos hipertensos. O exercício mostrou-se eficaz na redução do colesterol total e da lipoproteína de baixa densidade (LDL-c) e no aumento da lipoproteína de alta densidade (HDL-c). Além disso, apresentou efeitos benéficos na manutenção da capacidade funcional e da autonomia física, com importante impacto positivo na saúde e na qualidade de vida dos idosos.

Nesse sentido, a prática de atividades físicas reduz não apenas os sintomas depressivos, como também contribui para redução do risco cardiovascular. Aliado a essas práticas, é indispensável a associação da alimentação balanceada, rica fibras, vegetais e frutas, com ingestão equilibrada de lipídeos insaturados e evitar os saturados. Por essa razão, a associação dessas práticas deve ser estimulada para todos os pacientes com doenças cardiovasculares, independentemente da existência de depressão (DALMAZO AL, et al., 2019; LARKIN E e WHOOLEY MA., 2013).

Mais especificamente, o treinamento aeróbico de intensidade moderada mostrou bons resultados ao agir na permuta do colágeno, reduzindo o estresse oxidativo mitocondrial e o modelamento cardíaco de ventrículo esquerdo em camundongos ooforectomizadas a partir da desaceleração da apoptose de fibras musculares (MENDES F e MARTINS D, 2020).

Devido a esses dados, as atuais Diretrizes Europeias e Americanas recomendam a realização rotineira de uma triagem para depressão nos pacientes com doença cardiovascular. Caso diagnosticada, uma abordagem conjunta das duas doenças, através de terapias farmacológicas e não farmacológicas, deve ser realizada. No que se refere ao desfecho cardiovascular do paciente, o tratamento da depressão e ansiedade com terapia cognitivo-comportamental (TCC) não influenciou na ocorrência de eventos a longo prazo, enquanto o tratamento farmacológico com antidepressivos se mostrou efetivo (BUCCIARELLI V, et al., 2020).

Em contrapartida ao possível efeito de estímulo à agregação plaquetária nos transtornos mentais, o uso de antidepressivos, em especial os Inibidores Seletivos da Recaptação de Serotonina (ISRS), diminuem a atividade plaquetária e sua agregação, podendo contribuir para reduzir o risco de eventos cardiovasculares futuros, ao exemplo dos antiagregantes plaquetários (LARKIN E e WHOOLEY MA., 2013). 
A terapia farmacológica, também resultou em redução significativa em marcadores de ativação plaquetária/endotelial, como o fator plaquetário e $\beta$-tromboglobulina, em marcadores inflamatórios, como fator de necrose tumoral- $\alpha$ e proteína $C$ reativa, além de melhorar a variabilidade da frequência cardíaca (STEVEN MB e JOHN SR, 2015).

Almeida OP, et al. (2019), por outro lado, observou em seu estudo que tanto a depressão quanto o uso de antidepressivos aumentaram o risco cardiovascular em homens idosos. No entanto, afirma que o uso de antidepressivos reduz o risco cardiovascular nos pacientes com depressão, mas não naqueles sem a doença.

Nessa visão, pesquisas futuras poderiam focar nas nuances da relação entre situações estressantes e síndromes psiquiátricas em desfechos cardiovasculares a médio e longo prazo a partir de ensaios clínicos focados em superar as lacunas atuais sobre o assunto.

\section{CONSIDERAÇÕES FINAIS}

A revisão da literatura evidenciou a estreita relação entre os fatores psicológicos e emocionais e o surgimento de doenças cardiovasculares, confirmada por diversos estudos observacionais. Dentre os principais mecanismos fisiopatológicos descritos, os mais significativos são a privação do sono, o excesso de catecolaminas por ação do sistema nervoso simpático, a ativação do sistema renina-angiotensinaaldosterona, os distúrbios da homeostase e o estímulo à aterosclerose. A baixa adesão a tratamentos medicamentosos também foi evidenciada. Esses resultados contribuíram para fomentar novas estratégias no âmbito da abordagem dos pacientes ambulatoriais em geral que apresentam queixas de síndromes psíquicas, buscando evitar evolução de uma possível síndrome cardiovascular por meio da indicação precoce do exercício físico aeróbico e da alimentação balanceada, além de terapias medicamentosas.

\section{REFERÊNCIAS}

1. ALBUS C, et al. Significance of psychosocial factors in cardiology: update 2018: Position paper of the German Cardiac Society. Clinical research in cardiology: official journal of the German Cardiac Society, 2019; 108(11): 1175-1196.

2. ALMEIDA OP, et al. Depression, antidepressants and the risk of cardiovascular events and death in older men. Maturitas, 2019; 128: 4-9.

3. ANTUNES J. Longas jornadas de trabalho: efeitos na saúde. Psic., Saúde \& Doenças [Internet], 2020; 21(2): 311-321

4. BRASIL. Ministério da Saúde. Use o coração para vencer as doenças cardiovasculares": 29/9 - Dia Mundial do Coração. 2020. Disponível em: http://bvsms.saude.gov.br/ultimas-noticias/3305-use-o-coracao-para-vencer-as-doencascardiovasculares-29-9-dia-mundial-do-coracao. Acesso em 28 abr. 2021.

5. BUCCIARELLI V, et al. Depression and cardiovascular disease: The deep blue sea of women's heart. Trends Cardiovasc Med., 2020; 30(3): 170-176.

6. CASSIANO AN, et al. Efeitos do exercício físico sobre o risco cardiovascular e qualidade de vida em idosos hipertensos. Ciênc. saúde coletiva [Internet], 2020; 25(6): 2203-2212.

7. CHRISTIAN TMD. Clinical Features and Outcomes of Takotsubo (Stress) Cardiomyopathy. N Engl J Med. 2015. 373:929-938

8. COUTO HA, et al. Estresse ocupacional e hipertensão arterial sistêmica. Rev Bras Hipertens., 2007; 14(2): 112-115.

9. DALMAZO AL, et al. Stress and Food Consumption Relationship in Hypertensive Patients. Arq. Bras. Cardiol. [Internet], 2019; 113(3): 374-380.

10. FROMMEYER G, et al. Panic attacks and supraventricular tachycardias: the chicken or the egg?. Neth Heart J., 2013; 21 : 74-77.

11. GHADRI JR, et al. International Expert Consensus Document on Takotsubo Syndrome (Part I): Clinical Characteristics, Diagnostic Criteria, and Pathophysiology. European Heart Journal, 2018; 39(22): 2032-2046.

12. JEE YH, et al. Cohort study on the effects of depression on atherosclerotic cardiovascular disease risk in Korea. BMJ Open, 2019; 9(6): e026913.

13. KOMORI T, et al. Social status and modern-type depression: A review. Brain and behavior, 2019; 9(12): e01464.

14. LAMPERT R. Behavioral influences on cardiac arrhythmias. Trends in Cardiovascular Medicine, 2016; 26(1): 68-77.

15. LARKIN E, WHOOLEY MA. Depression and Cardiovascular Disease. Progress in Cardiovascular Diseases, 2013; 55(6): 511523.

16. LOURES DL, et al. Estresse Mental e Sistema Cardiovascular. Arq. Bras. Cardiol. [Internet], 2002; 78(5): 525-530.

17. MENDES F, MARTINS D. The Impact of Exercise On Inflammation, Oxidative Stress And Remodelling In Cardiac Muscle. Arq. Bras. Cardiol. [Internet], 2020; 114(1): 106-108.

18. OMS. ORGANIZAÇÃO MUNDIAL DA SAÚDE. Dia Mundial da Saúde Mental: uma oportunidade para dar o pontapé inicial em uma grande escala de investimentos. $2020 . \quad$ Disponível em: https://www.paho.org/bra/index.php?option=com_content\&view=article\&id=6263:dia-mundial-da-saude-mental-umaoportunidade-para-dar-o-pontape-inicial-em-uma-grande-escala-de-investimentos\&ltemid=839. Acesso em 28 abr. 2021.

19. RAJAN S, et al. Association of Symptoms of Depression with Cardiovascular Disease and Mortality in Low, Middle, and High Income Countries. JAMA Psychiatry, 2020; 77(10): 1052-1063.

20. SCHMIDT K, et al. Um Olhar sobre o Stress nas Mulheres com Infarto Agudo do Miocárdio. Arq. Bras. Cardiol. [Internet], 2020; 115(4): 649-657.

21. STEVEN MB, JOHN SR. Depression and cardiovascular disease. Trends in Cardiovascular Medicine, 2015; $25(7): 614-622$.

22. VELOSO MAOS, et al. Prevalence of depression and anxiety and their association with cardiovascular risk factors in Northeast Brasil primary care patients. Rev. Assoc. Med. Bras. [Internet], 2019; 65(6): 801-809. 